\begin{abstract}
ARTICULO
Revista Derecho - Año 3 edicion 5189 - 198

Web: http://revistas.unap.edu.pe/rd E-mail.com: revistaderecho@unap.edu.pe ISSN 2313-6944

\section{FACTORES PSICOLOGICOS QUE INFLUYEN EN LA COMISION DEL DELITO DE ESTAFA Y FALSIFICASION DE DOCUMENTOS DESDE LA CRIMINOLOGIA PSICOLOGICA}

\author{
CALLO CHAVEZ, Jhon Yehefer \\ HUAYHUA COLCA, Iván Victor \\ MAMANI QUISPE, Toribio Washington \\ URBINA VELEZ, Boney Well
}

\section{INFORMACION DEL ARTICULO \\ Art. Recibido: $18 / 03 / 19$ \\ Art. Aceptado: 25/03/19 \\ Art. Publicado: 15/04/19 \\ Criminología \\ Psicología criminal \\ Factores Psicológicos \\ Asesor:}

PALABRAS CLAVE:

Abg. Michael Espinoza Coila

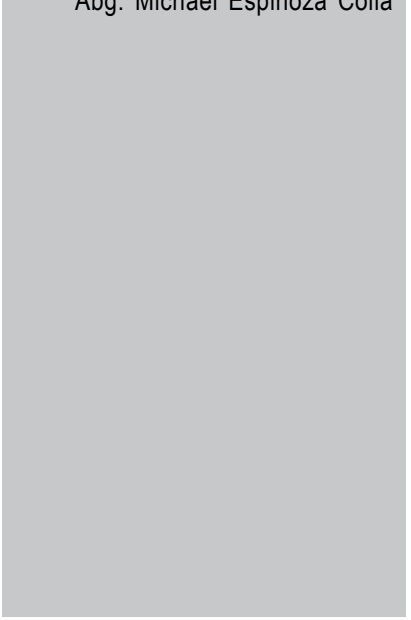

\section{RESUMEN}

El presente trabajo tiene como objetivo analizar y explicar los factores psicológicos para la comisión del delito de falsificación de documentos como fenómeno criminológico, de esta manera se busca un análisis y estudio desde un punto de vista de la escuela psicológica; de esta manera nos abocaremos a un expediente técnico factico, así también es indudable acudir a autores reconocidos que estudian sobre el tema antes planteado, así tenemos a Eugenio Raúl Zaffaroni en su libro «La cuestión Criminal», Luis Rodríguez Manzanera en su texto «Criminología» en donde hace más ahínco en la criminología y demás ramas, Alessandro Baratta que principalmente da una visión general del rema en su libro «Criminología Crítica y Crítica del Derecho Penal» y entre otros autores que estudiaremos para poder desarrollar de manera entendible el presente artículo.

De esta manera, en primer lugar se desarrollara sobre un tema netamente académico o doctrinario si se quiere llamar así, un punto muy importante para empezar el estudio es acerca de la criminología, su conceptualización así como de su concepto tradicional y un concepto científico de este tema relativamente nuevo; por otro lado también es importante analizar sobre si la escuela psicológica tradicional es suficiente para aportar a la Criminología o es que este tendría que tener una nueva visión desde su perspectiva, es decir, si se necesita una nueva psicología para el estudio del delincuente o del delito.

En segundo lugar, haremos hincapié en un análisis factual sobre el tema, esto tomando en cuenta el expediente lo cual guardaremos reserva de los datos respectivos. Por último, se llegará a la conclusión del aporte de la Psicología para el estudio de la Criminología y este como incidió en el caso factual antes ya mencionado.

chaverjhon@gmail.com

buaybua25@gmail.com

washingtonwm@gmail.com

urbina23@gmail.com
\end{abstract}




\section{PSYCHOLOGICAL FACTORS THAT INFLUENCE IN THE COMMISSION OF THE CRIME OF SCAM AND DOCUMENT FALSIFICATION FROM PSYCHOLOGICAL CRIMINOLOGY}

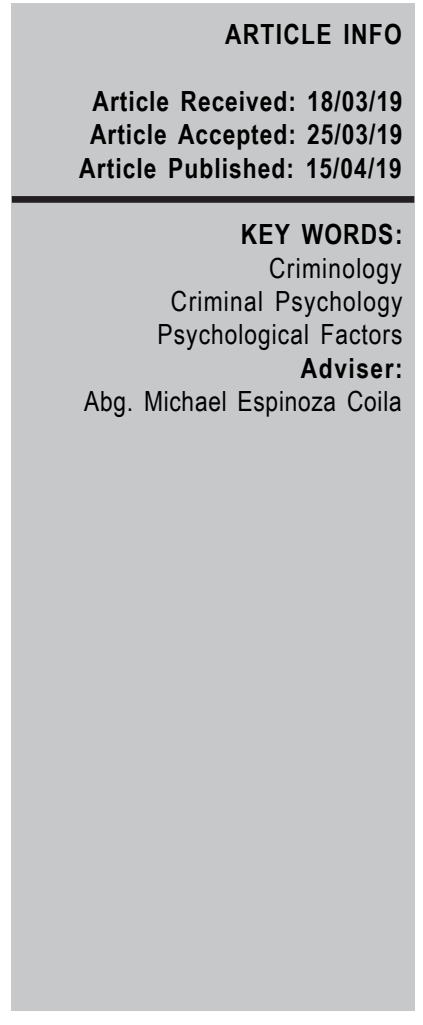

\section{ABSTRACT}

This work has as objective to analyze and explain the psychological factors for the commission of the crime of falsification of documents as the criminological phenomenon, in this way an analysis and study is sought from a psychological school point of view; In this way, we will engage in a technical file, as well as undoubted. The authors acknowledge the subject. Before the proposal, and also Eugenio Raúl Zaffaroni in his book «The criminal question», Luis Rodríguez Manzanera in his text «Criminology» where he is more committed to criminology and other branches, Alessandro Baratta, who mainly in an overview of the remake in his book «Criminology Criticism and Criticism of Criminal Law» and among other authors who study the power to understand the present article in an understandable way. In this way, in the first place, it will be developed on a purely academic or doctrinal subject if it is to call it that, a very important point to start the study is about criminology, its conceptualization as a traditional concept and a scientific concept of this shared topic new; On the other hand it is also important to analyze the traditional psychological school is enough to contribute to Criminology or to take into account the new psychology for the study of the offender or crime Secondly, we will focus on an analysis made on the subject, this taking into account the file what we reserve the relevant data. Finally, consult the conclusion of the contribution of Psychology for the study of Criminology and in this way.

190 Revista Derecho - 5 (2019) 
ANALISIS ACADEMICO DE LA CRIMINOLOGIA Y SU INTERRELACION CON LA DISCIPLINA PSICOLOGICA

En el presente esquema, se analizará un aspecto teórico de la Criminología y su interrelación con las demás ramas de conocimiento, pero de manera específica en la criminología psicológica. De este modo, es necesario abordar el concepto de este, así como el delito tipificado en el Código Penal vigente, así como su análisis doctrinario, lo cual se desarrolla en las siguientes líneas.

\section{CONCEPTO DE CRIMINOLOGIA}

Desde un punto de vista psicológico, según Vásquez Cigarroa (2015), la criminología es una ciencia inter y multidisciplinaria cuya finalidad es el estudio, análisis, intervención, prevención y profilaxis de tanto el crimen, como el criminal y la criminalidad. Por tanto, el objeto de estudio de la criminología sería la conducta antisocial, aquellas conductas que se desvían de lo normalmente aceptado por la sociedad y que incluso atenta contra la integridad de esta, partiendo de la premisa de que el hombre es un ser biopsicosocial y, por ende, el delito como conducta humana debe explicarse como un fenómeno que abarca aspectos biológicos, psicológicos y sociológicos.
De esta manera, podemos señalar que el estudio de la criminalidad humana es un fenómeno complejo, por lo que nos atrevemos a decir que cada día es más amplio, también cabe señalar que sobre el tema planteado existen diversas y diferentes ciencias que han aportado sus conocimientos y teorías explicativas para poder lograr una mejor comprensión, evaluación y tratamiento de los delincuentes, así como de su erradicación. En ese entender una de estas ciencias ha sido la psicología, y dentro de ésta, la psicología criminal

Por tanto, y según afirma Nicolás Guardiola (2006), «la psicología criminal sería una ciencia social aplicada, que busca resolver problemas complejos, utilizando un método y no actúa de forma aislada, ya que es interdisciplinar»

\section{LA CRIMINOLOGIA Y OTRAS DISCIPLINAS}

Para analizar el objeto de estudio, nos apoyaremos en uno de los profesores que ha desarrollado textos acerca del tema, en ese entender García Álvarez (2017) sostiene que para abordar el tema de los factores que influyen en la comisión de un delito, es necesario hacerlo desde lo interdisciplinario, multidisciplinario y transdisciplinario. Cuando todas las ciencias se reúnen alrededor del objeto de estudio, en este caso la delincuencia, estaremos hablando de constelación criminológica, y dentro de ésta vemos en primer lugar que la

Revista Derecho - 5 (2019) 191 
investigación científica interdisciplinaria, ayuda porque las disciplinas se entrelazan para estudiar el mismo objeto de estudio, tienen el mismo nivel y son de la misma rama de estudio. Como ejemplo de éstas, son la Psicología, Sociología, Derecho, Antropología, Política, Economía. De este modo, en el presente trabajo pues sin duda abarca la rama de la Psicología

Siguiendo la misma línea, según señala Howitt (2012), la psicología criminal, aunque tiene un desarrollo reciente, ha pasado a convertirse en uno de los aspectos más populares que existen en la psicología recientemente. Dependiendo de la orientación teórica de quiénes formulen una definición del concepto de psicología criminal, existen numerosas definiciones, ya que, la Psicología no es una ciencia compacta que incluya una sola teoría o aproximación en su estudio. Atendiendo a su origen etimológico, la psicología criminal podría entenderse como el estudio del alma del criminal, pero en este caso atendería a su personalidad.

Nuestro autor resalta que, más concretamente, la psicología criminal puede definirse desde una perspectiva más amplia o más estrecha, y dependiendo de ésta, incluirá unos u otros aspectos. La definición estrecha sugeriría que la psicología criminal concierne a todos los aspectos de la psicología del ofensor, el problema que ésta presenta, es que parece que se centra solamente en el cri- minal, pero también debería incluir los aspectos psicológicos del hecho criminal acaecido, ya que la criminalidad no es una característica de los individuos que pueda ser separada del contexto social del crimen y del sistema de justicia criminal

En ese sentido, consideramos que esta definición más amplia y no restrictiva del tema, permite incluir en la presente disciplina, además del estudio de la conducta del delincuente, el análisis de todo lo que rodea al hecho criminal. Tanto el delincuente en concreto, las motivaciones y sus factores tanto endógenos como exógenos. Por tanto, la psicología criminal estudiaría al delincuente, el hecho criminal e intentaría prevenir el delito mediante programas de tratamiento a los delincuentes y medidas para hacer menos vulnerables a las víctimas (Velazco Dias, 2014).

Por tanto, y a manera de resumen, según afirma Nicolás Guardiola (2006), «la psicología criminal sería una ciencia social aplicada, que busca resolver problemas complejos, utilizando un método y no actúa de forma aislada, ya que es interdisciplinar»

\section{EL ESTUDIO PSICOLOGICO DE LA CRIMINOLOGIA EN EL DELITO DE ESTAFA}

Es importante tener una idea acerca del delito de estafa, de esta manera, consideramos que la estafa es, ante todo, 
un delito contra intereses patrimoniales, esto entendido tanto en la afectación de intereses patrimoniales individuales y colectivos.

De este modo, Villavicencio Terreros (2006), señala que el delito de estafa consiste en el empleo de artificio o engaño, a fin de procurar para sí o para terceros un provecho patrimonial en perjuicio ajeno. El delito de estafa es una forma de defraudación, vale decir, la defraudación en el género y la estafa, una de sus modalidades típicas. En suma, la estafa es la conducta engañosa, con ánimo de lucro injusto, propio o ajeno, que habiendo determinado un error en una o varias personas, les induce a realizar un acto de disposición, consecuencia del cual en un perjuicio en su patrimonio o en el de un tercero.

Por otra parte, en palabras de Muñoz Conde, podemos señalar «Que sobre la estafa define, que lesiona, al mismo tiempo, la buena fe o las relaciones fiduciarias que surgen en el tráfico jurídico. Normalmente se espera que se cumplan las obligaciones contraídas y que sí, por ejemplo, se compra un kilo de pan sea efectivamente un kilo y, además, de pan. Pero si la sustancia o cantidad del objeto comprado no corresponde a lo pactado, se frustra una legítima expectativa que debe ser protegida de algún modo, para asegurar y garantizar un normal tráfico económico.
Ahora bien, en palabras de Rechea Alberola (2018), señala que aunque la finalidad político criminal perseguida con la tipificación del delito de estafa sea ésta, el delito como tal se castiga en tanto lesiona un derecho patrimonial individual. Este contenido patrimonial de la estafa no debe ser olvidado, para no castigar indebidamente hechos que frustran expectativas de comportamiento en el tráfico jurídico económico, pero que no producen perjuicios económicos para nadie en concreto.

De lo señalado líneas arriba y conforme la doctrina y las normas punitivas, podemos afirmar que el delito de estafa es un tipo doloso, pues el agente sabe, idea las consecuencias que la acción va arraigar, en ese sentido, pues se cumple con todas las fases del iter criminis.

\section{SU TIPIFICACION EN EL} CODIGO PENAL VIGENTE

$$
\text { Según Bramont Arias-Torres }
$$
(2001), señala que «los delitos de falsificación material de documentos son, en general, tipos delictivos con estructuras típicas muy complejas. De ahí que el estudio sobre estos sea objeto de un amplio debate doctrinario. Amén de ello es, además, uno de los delitos de más común realización que constituye un gran porcentaje de causas que se tramitan en el Poder Judicial»

Revista Derecho - 5 (2019) 193 


\section{* Casos de defraudación}

Artículo $197^{\circ}$. - La defraudación será reprimida con pena privativa de libertad no menor de uno ni mayor de cuatro años y con sesenta a ciento veinte días-multa cuando:

1. Se realiza con simulación de juicio o empleo de otro fraude procesal.

2. Se abusa de firma en blanco, extendiendo algún documento en perjuicio del firmante o de tercero.

3. Si el comisionista o cualquier otro mandatario, altera en sus cuentas los precios o condiciones de los contratos, suponiendo gastos o exagerando los que hubiera hecho.

4. Se vende o grava, como bienes libres, los que son litigiosos o están embargados o gravados y cuando se vende, grava o arrienda como propios los bienes ajenos.

\section{* Falsificación de documentos}

Artículo $427^{\circ}$.- El que hace, en todo o en parte, un documento falso o adultera uno verdadero que pueda dar origen a derecho u obligación o servir para probar un hecho, con el propósito de utilizar el documento, será reprimido, si de su uso puede resultar algún perjuicio, con pena privativa de libertad no menor de dos ni mayor de diez años y con treinta a noventa díasmulta si se trata de un documento público, registro público, título auténtico o cualquier otro trasmisible por endoso o al portador y con pena privativa de libertad no menor de dos ni mayor de cuatro años, y con ciento ochenta a trescientos sesenticinco días-multa, si se trata de un documento privado.

El que hace uso de un documento falso o falsificado, como si fuese legítimo, siempre que de su uso pueda resultar algún perjuicio, será reprimido, en su caso, con las mismas penas.

FACTORES PSICOLOGICOS A CONSIDERAR EN EL DELITO DE ESTAFA

Para este tópico, consideramos la entrevista realizada a la Psicóloga Irma Cuba Valencia y a la Lic. Luz Díaz Polanco, en donde textualmente sostienen que, a su juicio, los principales factores psicológicos que se debe tener para que una persona cometa un delito tal es el caso de falsificación de documentos y estafa son los siguientes:

* E1 comportamiento: Es entendida como es el conjunto de respuestas, bien por presencia o por ausencia, que presenta un ser humano o hasta animal, en relación con su entorno o1mundo de estímulos. El comportamiento puede ser consciente o inconsciente, voluntario o involuntario, etc. según las circunstancias que lo afecten.

194 Revista Derecho-5 (2019) 
* La insatisfacción: Entendemos por esto que las personas no somos una especie conformista, siempre buscamos algo más de lo que realmente se necesita, es por ello que esa insatisfacción haga que una persona saque provecho de otras circunstancias.

* El egocentrismo: Se entiende como egocentrismo al amor excesivo que una persona tiene sobre sí misma, el cual la lleva a atender solamente su propio interés, sin interesarse por el bienestar ajeno.

* Los trastornos personales: Esto hace referencia a que las personas en etapas pasadas sufrimos algún tipo de trastorno, esta puede ser por violencia familiar o bien algún abuso sufrido.

Los factores señalados líneas arriba son los que principalmente se presentan en el sujeto activo para que tenga un cambio repentino a lo que le llevara a cometer el delito que posteriormente con el desarrollo del tema se irá esclareciendo. Con lo anterior desarrollado, posteriormente se abordará lo que es el análisis del expediente con el que se analizara estos factores psicológicos.

\section{ANALISIS FACTUAL DE LA PSI- COLOGIA CRIMINAL EN EL EXPDIENTE No054-2016-INPE/ 24-803.SCTP}

Con las bases doctrinarias desarrolladas líneas arriba, ello nos ayudara a interpretar y entender mejor el expediente para así poder analizar si los factores que se hallaron son verdaderamente lo que conlleva a la comisión de delitos, tales como la falsificación de documentos y estafa

\section{MOTIVACIONES PARA LA REALIZACION DEL DELITO}

Con fines académicos, reservaremos el nombre real de la persona condenada por este delito, en ese entender, solo para tener una referencia académica lo llamaremos con las iniciales C.V.M. De esta manera, en el expediente que se está analizando, desarrollaremos los hechos de manera breve para llegar al objetivo del trabajo, que es analizar los factores psicológicos que influyeron en el sujeto activo

En ese entender, la persona C.V.M. mediante engaño y astucia le pide a la señora Rosa los documentos de su bien inmueble para que posteriormente este lo falsifique dichos documentos a su favor, en ese sentido, el sujeto activo, en el expediente en análisis señala que precisamente era su ambición lo que le llevo a cometer el delito, esto en sus declaraciones del informe psicologico en donde textualmente señala que: «... yo quería tener más dinero, porque actualmente en mi trabajo me pagan muy poco, es por eso que vi la oportunidad de ganar dinero, en donde

Revista Derecho - 5 (2019) 195 
a la Sra. Rosa le pedí la suma de treinta mil soles $(\ldots) »$

En ese entender aquí se presenta uno de los factores psicológicos antes señalados, es decir, sale a brote la insatisfacción del individuo, lo cual lo llevo a cometer dicho delito, de este modo, también se considera los trastornos personales, que según C.V.M. en su infancia presenciaba constantemente agresiones físicas y verbales en su familia.

ESTADO DE SALUD MENTAL ANTES DE COMETER EL DELITO

Muchas veces existe amplio debate acerca de este tópico, pues existe una parte de la sociedad que considera que el sujeto activo, es decir, la persona que comete el delito ya no es alguien que puede ser aceptado por la sociedad e incluso sostienen que el delincuente nace. Sin embargo, existe otra parte de la población, quizás mínima, que considera que la persona que comete el delito puede readaptarse a la sociedad, esto cumpliendo con los fines del derecho penal.

De este modo, en el presente trabajo no se busca explicar el estado de salud mental antes de la comisión de cualquier otro delito, sino se abarca acerca del expediente en análisis y el delito de falsificación de documentos y estafa.

196 Revista Derecho - 5 (2019)
Según el informe Psicológico 0392016-INPE, señala que el sentenciado gozaba de una personalidad tranquila, atenta y con ganas de sobresalir en su vida diaria. En es entender, consideramos que necesariamente esos factores anteriormente señalados influyen bastante para que la persona cambie de estado de ánimo y cometa ese delito.

\section{¿SE PUEDE CAMBIAR EL ESTA- DO DE SALUD MENTAL DES- PUES DE COMETER EL DELI- TO?}

Siguiendo las líneas del Informe Psicológico, sostiene que la persona si puede cambiar su estado de salud mental para su desenvolvimiento en la sociedad. De esta manera, el informe señala que «el interno aprendió que es importante reconocer que amistades son positivas para su crecimiento personal, desarrolla y fortalece competencias sociales que le permitan la reducción de probabilidad de reincidencia delictiva. Hoy en día ha desarrollado más independencia y estabilidad emocional. Por otro lado, considera que a través del trabajo se obtienen logros personales» De lo señalado, resaltamos que en el expediente que se analiza, el sujeto activo si pudo rehabilitarse después de haber cometido el delito, sin embargo, recalcamos que este no es un delito fatídico, es decir, no se trata de homicidio, lo cual consideramos que, si se tratase de este tipo de delitos, a lo mejor 
los resultados no serían los mismos, pues una es cometer el delito de homicidio y otra muy distinta al de falsificación de documentos.

\section{CONCLUSION}

Consideramos que una persona antes de cometer el delito, tal es el caso de estafa y falsificación de documentos, la persona ya ha ideado lo que va hacer, pues esto se trata de un delito doloso, en donde el agente sabe las consecuencias que puede acarrear, es decir, tiene toda la intención de realizarlo y lo hace de manera consiente.

Por otro lado, consideramos que los factores de egocentrismo, insatisfacción y los trastornos personales son factores que necesariamente se presentan para que el agente pueda realizar el delito, pues mucho depende de ello para que cambie su conducta, tal es el caso en el expediente que se analizó, pues el interno señala que llevaba una vida normal y que por la insatisfacción de su remuneración lo llevo a realizar el delito.

También cabe considerar que el estado de salud mental de la persona que cometió el delito puede mejorar, pero siempre y cuando el agente tenga la intención de hacer un cambio a su conducta, sin embargo, consideramos que no siempre una persona puede cambiar su pensamiento o conducta pues muchas veces se ve que solamente se finge un cambio en el momento del informe psicológico con el fin de que se le disminuya la pena.

\section{REFERENCIAS}

Bramont Arias Torres, L. A. (2001). Manual de Derecho Penal Parte Especial. Lima: Editorial San Marcos.

Garcia Alvarez, F. (2017). Constelacion Criminologica. Mexico: Sociedad Mexicana de Criminologia.

Howitt, D. (2012). Que es la Psicologia Criminal y Forense. Mexico: Editorial Porrua.

Rechea Alberola, C. (2018). Manuales de Criminologia. España: Editorial Sintesis.

Redondo Illescas, S. (2007). La Psicologia de la Delincuencia. Barcelona: Seccion Monografica.

Vasques Cigarroa, R. (2015). Criminologia Psicologica. Mexico: Porrua.

Velazco Dias, C. (2014). La Psicologia Aplicada en la Investigacion Criminal. Colombia: Revista Electronica Penal.

Villavicencio Terreros, F. (2006). Derecho Penal Parte Especial. Lima: Grijley. 
198 Revista Derecho - 5 (2019) 